\title{
«Schönheit hat viel mit Ausdruck zu tun»
}

\section{Daniel Lüthi}

Text und Bilder

danielluethi@gmx.ch
Dies gleich vorweg: Ja, sie ist schön. Aber: Ihre Schönheit lässt sich weder auf die gängigen Klischees reduzieren, noch auf die vier Zahlen, die diese Schönheit quasi amtlich beglaubigen. 179 (die Grösse) und 86-62-91 (der Umfang von Brust, Taille und Gesäss) so wird Simone in diesen Tagen angepriesen. Als Kandidatin Nummer 4 einer Wahl, die sie zur Nummer 1 machen könnte, zur «Miss Schweiz 2011». Schön, wenn es so käme. Denn Simone Casanova ist nicht bloss eine schöne junge Frau, sondern eine erstaunlich reife, schöne Persönlichkeit.

\section{Die Kandidatur}

«Zurzeit lebe ich in drei Welten», sagt sie, «und ich versuche, sie alle möglichst gut zu managen: die Kandidatur, die Arbeit und das Privatleben.» Im Vorder- grund steht momentan sicher die Kandidatur. «Sie verfolgt mich seit Mitte Februar, und ich kann sie jetzt nicht einfach abstellen. Aber ich habe das Glück, dass ich dies alles sehr schätze und geniesse.» Dies alles: Das ist Glitzer und Glamour, Make-up und Show-off. Das sind Video-Drehs, Foto-Shootings, InterviewSessions und Laufsteg-Walks. Ein Hauch Showbusiness, ein Touch Prominenz. Geschenktes Glück für eine beschränkte Zeit. «Eine weitere Lebenserfahrung», fasst Simone zusammen, «die Möglichkeit, mich selber zu reflektieren - zu schauen, wie ich mich gebe, und zu merken, wer ich bin. Wichtig ist, dass ich mich selber bleibe, und dass auch meine Freunde stets sagen können: Das ist die Simone, die wir kennen.»

Erfahrungen als Model hat sie nicht - und gäbe es diese Kandidatur nicht, sie würde es auch nicht unter

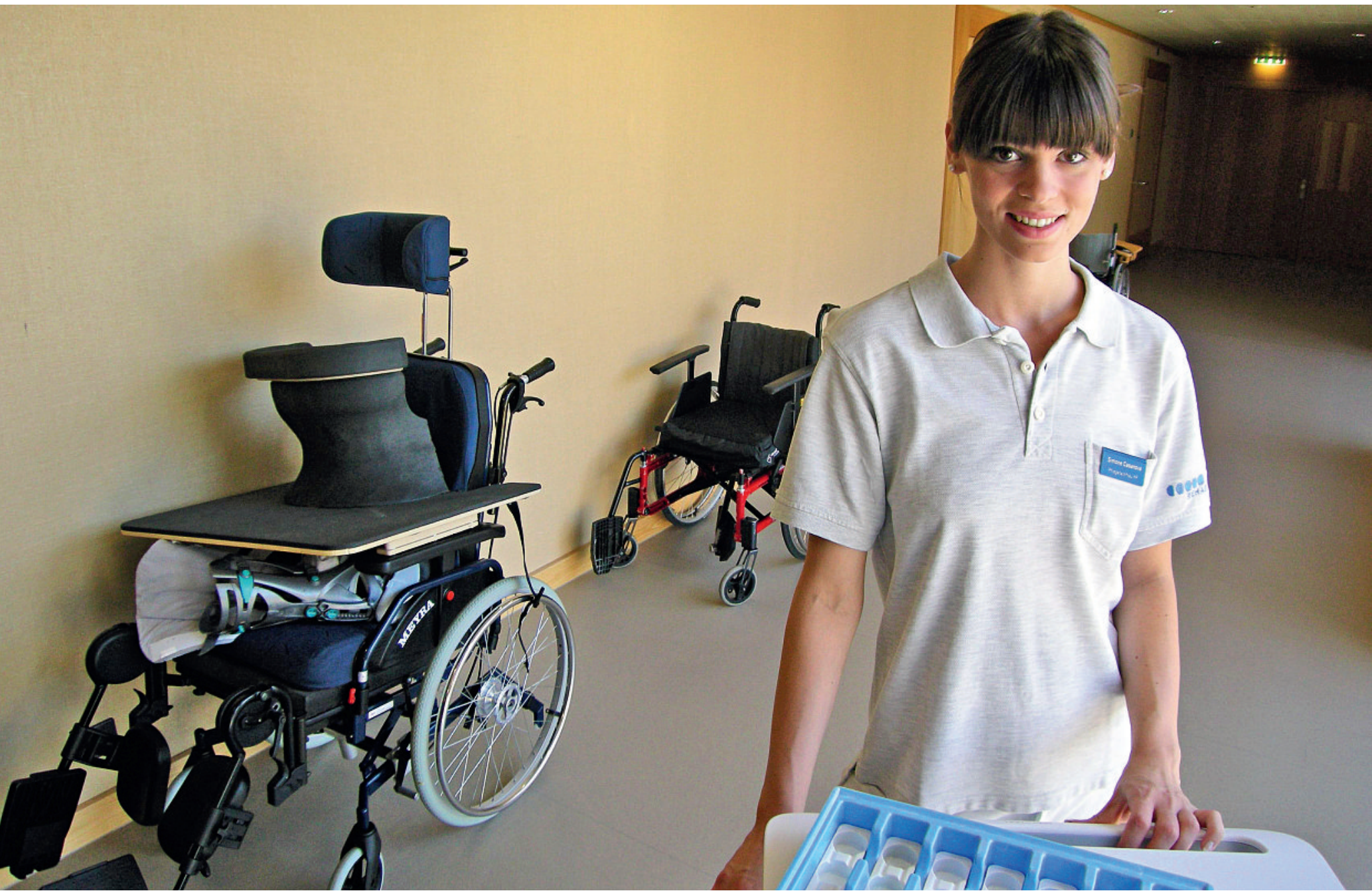


allen Umständen werden wollen. Eine SchönheitsOperation für sich schliesst sie - vorläufig zumindest - aus. Dagegen signalisiert sie Verständnis für die ästhetische Chirurgie in gewissen Situationen: «Eine Bekannte wollte eine Brustvergrösserung. Ich habe sie unterstützt, weil sie sich in ihrem Körper nicht wohl fühlte. Aber man muss sich auch vor falschen, ja fahrlässigen Versprechungen hüten. Und man muss wissen, dass eine solche Operation immer auch ein Eingriff ist, der Risiken birgt.»

Und die Schlankheit, ja die Magersucht als Schönheitsideal? «Ich habe immer normal gegessen. Gleichzeitig blieb ich immer schlank. Mit 12 war ich deshalb «der Storch». Mit 17 wurde daraus «eine grosse, schlanke, junge Dame. Im Übrigen hat sich die Model-Welt gewandelt. Heute ist Grösse 36 oder 38 auch möglich.» Wieder blickt sie mit ihren grossen, braunen Augen in die Ferne. «Nun ja», fügt sie bei, «ein Laufsteg in New York, Mailand oder Paris, das ist eine andere Sache.»

\section{«Wichtig ist, dass ich mich selber bleibe.»}

\section{Das Rampenlicht}

Im Juni war das Missen-Camp in Zermatt. Die Konkurrentinnen wurden zum Teil zu Freundinnen. Vor allem aber wurden sie mit der Organisation und den Regeln des Spiels vertraut gemacht: wann welchen Schmuck und welche Kleider tragen, wann welche Ansprüche der Sponsoren erfüllen, wie gehen, wie stehen, wie agieren, wie dreinschauen - das waren Themen. Auch im 2. Missen-Camp in Lugano, wo der grosse Auftritt am grossen Tag geübt wurde.

Es war Simones etwas älterer Bruder, der sie motiviert hat, mitzumachen. «Du bist gross und schlank», habe er gesagt, «aber du bist mehr als ein Model. Versuche es!» Eine Herausforderung sei das Ganze, sagt Simone Casanova immer wieder. Zum Beispiel wolle sie herausfinden, ob diese Welt wirklich so oberflächlich sei, wie sie in den Medien immer wieder beschrieben werde. Neugier also. Und die Lust auf Abenteuer, auf eine Selbst-Erfahrung der besonderen Art.

Auch ihr Freund habe ihr Mut gemacht, erzählt Simone, ja er habe ihr sogar vorgemacht, wie man einen Traum leben könne: «Er hat sich auch mal ausgeklinkt und hat viel investiert, um in Neuseeland auf professionellem Niveau Fussball spielen zu können.»

Ein Schritt wie der ihre ist ein Wagnis, dessen ist sich Simone Casanova bewusst: «Das Rampenlicht kann auch zur Belastung für eine Beziehung werden.» Mehr noch: Wer sich dem Urteil der Öffentlichkeit aussetzt, ist verletzlich. Es besteht die Gefahr, manipuliert oder gar missbraucht zu werden. «Bisher

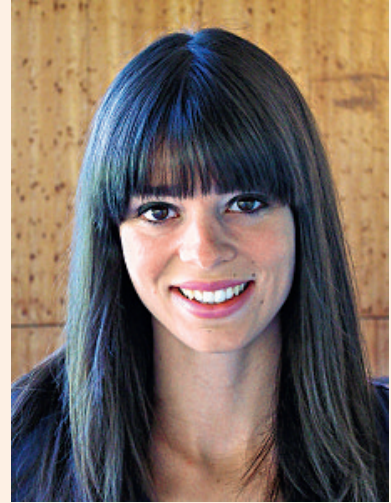

\section{Simone Casanova}

Simone Casanova wurde im September 1986 in Basel geboren. Sie wuchs in Pfeffingen und Therwil (BL) auf und besuchte dort auch die Schulen. Am Bethesda-Spital in Basel liess sie sich zur Pflegefachfrau HF ausbilden. 2007 machte sie das Diplom. Seit vier Jahren arbeitet sie in einem 100\%-Pensum auf der WachkomaStation der REHAB-Klinik Basel (Schweizerisches Paraplegikerzentrum), inzwischen auch als Ausbildnerin. Simones Vater Reto arbeitete als Drogist, Mutter Ruth war Praxisassistentin.

Sie starb 53-jährig an einem Hirntumor, noch vor Simones 18. Geburtstag.

Am 24. September 2011 steht Simone Casanova in Lugano auf der grossen Show-Bühne: Als älteste von 12 jungen Frauen nimmt die 25-Jährige an der diesjährigen Miss-SchweizWahl teil.

Simone Casanova lebt zusammen mit ihrem Bruder Sandro in Allschwil/BL.

konnte ich immer selber entscheiden, was ich anziehe und wie ich mich ablichten lasse», kommentiert Simone.

\section{Das Ereignis}

Die Show am Samstag, 24. September 2011, wird ein Ereignis sein, so oder so. «Ich freue mich sehr darauf, endlich diese Bühne betreten zu können. Über 20 meiner Leute werden im Publikum sitzen und mitfiebern.» Das Ereignis könnte den weiteren Weg von Simone Casanova innerhalb von Sekundenbruchteilen nachhaltig in eine völlig andere Richtung lenken: 
Falls sie gewinnt, wird sie mindestens ein Jahr lang ein komplett anderes Leben leben als bisher. Das mediale Leben einer Miss eben, das Leben als Schönheit. Mit Gala-Auftritten und Autogramm-Stunden in einer Welt von Glitzer und Glamour, von beautiful bodies mit strahlendem Lachen und finanzstarken Sponsoren mit eindeutigen Absichten.

Und falls sie die Krone nicht erhält, wird sie nächste Woche weiterarbeiten wie bisher.

In einem Rehabilitationszentrum für Querschnittgelähmte und Hirnverletzte. Auf einer Station, wo Menschen liegen, die dem Tod zeitweise wahrscheinlich näher sind als dem Leben. «Wachkoma» heisst dieser Zustand. Ein Ereignis hat diese Menschen hierhin katapultiert, hat ihren Weg innerhalb von Sekundenbruchteilen in eine völlig andere Richtung gelenkt. Das Ereignis war keine Wahl, sondern ein Schicksalsschlag. Ein Schädel-Hirn-Trauma oder ein Infarkt beispielsweise, ein Unfall oder eine schwere Krankheit mit einer sehr schweren Gehirnschädigung als Folge.

\section{«Wenn ich ein solches Leben nicht als lebenswert erachten würde, könnte ich nicht hier arbeiten.»}

Und jetzt - ein besonderer Moment - verbindet die junge Pflegefachfrau und Miss-Schweiz-Kandidatin ihre beiden Welten mit zwei Begriffen: «Schönheit», sagt die schöne Simone, «Schönheit hat viel mit Ausdruck zu tun.»

Pulsanstieg, Fieberschübe, Schwitzattacken oder Spasmen sind Ausdrucksformen hier. Zeichen schwerer funktionaler Störungen, aber auch kommunikative Möglichkeiten. «Im Durchschnitt bleiben die Leute etwa ein halbes Jahr bei uns auf der Station. Es kann aber auch sein, dass jemand erst nach 10 Jahren aus diesem Zustand erwacht, oder aber nie mehr bewusst am Leben teilnehmen kann.» Überleben also als Lebensform? «Wenn ich ein solches Leben nicht als lebenswert erachten würde, könnte ich nicht hier arbeiten», antwortet Simone.

Und wenn sie nicht hier arbeiten würde, könnte sie auch nicht so selbstverständlich sagen: «Ich setze mich intensiv mit Leben und Tod auseinander. Und ich glaube, dass mich verstorbene Verwandte und Bekannte abholen kommen und begleiten, wenn's bei mir einmal so weit ist. Für mich selber habe ich deshalb entschieden, dass ich - sollte es mich treffen nicht an eine Beatmungsmaschine angeschlossen werden möchte.» Klar: Eine entsprechende PatientenVerfügung ist unterschrieben - «eine solch schwierige Entscheidung möchte ich nicht meiner Familie aufbürden, die Situation wäre ohnehin schon herausfordernd genug», erklärt Casanova. Und ebenfalls klar: Wenn eine 25-Jährige so denkt und spricht, liegt dem eine tiefschürfende persönliche Erfahrung zugrunde.

\section{Die Mutter}

Als Simone 17 Jahre alt war, verlor sie ihre Mutter. Diese starb, 53-jährig, an einem Hirntumor. «Warum?», fragt Simone. Und antwortet gleich selber: «So lange man lebt, gibt es keine Antwort auf diese Frage.» Sie hat nach Interpretationen gesucht - und welche gefunden. «Jeder Mensch hat seine Aufgaben und seine Probesituationen, die er durchleben muss. Ich glaube, vieles ist vorbestimmt. Dies nimmt mir die Angst. Meine Mutter hat ihre Ziele erreicht. Deshalb konnte sie in ein anderes Leben übergehen. Für mich war ihr Tod kein Abschluss - sie ist immer noch da.»

Wie anders tönt jetzt plötzlich der Satz, mit dem Simone Casanova in der «Schweizer Illustrierten» als Miss-Schweiz-Kandidatin zitiert wurde: «Mama wäre stolz auf mich»?!

Wie unbedeutend ist Simones Aussage geworden, ihre Nasenlöcher seien vielleicht ein wenig zu gross?

Und wie sehr sind die drei Welten dieser jungen Frau auf einmal zu einer verschmolzen?!

\section{Die nächste «Begegnung mit ...»}

Einmal im Monat stellt die Schweizerische Ärztezeitung eine Persönlichkeit vor, die sich im Gesundheitswesen engagiert. Im Oktober schildert Daniel Lüthi seine Begegnung mit Heiner Frost, Clinical Reviewer beim Schweizerischen Heilmittelinstitut Swissmedic. 